\title{
Grid Occupancy Estimation for Environment Perception Based on Belief Functions and PCR6
}

\author{
Julien Moras, Jean Dezert, Benjamin Pannetier \\ The French Aerospace Lab, ONERA/DTIM/EVF, F-91761 Palaiseau, France.
}

\begin{abstract}
In this contribution, we propose to improve the grid map occupancy estimation method developed so far based on belief function modeling and the classical Dempster's rule of combination. Grid map offers a useful representation of the perceived world for mobile robotics navigation. It will play a major role for the security (obstacle avoidance) of next generations of terrestrial vehicles, as well as for future autonomous navigation systems. In a grid map, the occupancy of each cell representing a small piece of the surrounding area of the robot must be estimated at first from sensors measurements (typically LIDAR, or camera), and then it must also be classified into different classes in order to get a complete and precise perception of the dynamic environment where the robot moves. So far, the estimation and the grid map updating have been done using fusion techniques based on the probabilistic framework, or on the classical belief function framework thanks to an inverse model of the sensors. Mainly because the latter offers an interesting management of uncertainties when the quality of available information is low, and when the sources of information appear as conflicting. To improve the performances of the grid map estimation, we propose in this paper to replace Dempster's rule of combination by the PCR6 rule (Proportional Conflict Redistribution rule \#6) proposed in DSmT (Dezert-Smarandache) Theory. As an illustrating scenario, we consider a platform moving in dynamic area and we compare our new realistic simulation results (based on a LIDAR sensor) with those obtained by the probabilistic and the classical belief-based approaches.
\end{abstract}

Keywords: Grid map, cell occupancy, perception, belief functions, DSmT, PCR6, robotics.

\section{INTRODUCTION}

Occupancy Grids (OG) are often used for robot environment perception and navigation, which requires techniques for data fusion, ${ }^{1}$ localization ${ }^{2}$ and obstacle avoidance. ${ }^{3}$ As OGs manage a representation of the environment that does not make any assumption on the geometrical shape of the detected elements, they provide a general framework to deal with complex perception conditions. In our previous works, we did focus on the use of a multi-echo and multi-layer LIDAR system in order to characterize the dynamic surrounding environment of a robot navigating in an unrestricted area. The perception strategy involved map estimation and scan grids ${ }^{4,5}$ based either on the classical Bayesian framework, or on classical evidential framework based on Dempster-Shafer theory (DST) ${ }^{6}$ of belief functions. The map grid acts as a filter that accumulate information and allows to detect moving objects. A comparative analysis of performances of these approaches has already been published recently in. ${ }^{7}$

In dynamic environments, it is crucial to have a good modeling of the information flow in the data fusion process in order to avoid adding wrong implicit prior knowledge that will need time to be forgotten. In this context, evidential OG are particularly interesting to make a good management of the information since it is possible to explicitly make the distinction between non explored and moving cells. In this paper, we explore the use of Dezert-Smarandache Theory ${ }^{8}$ (DSmT) as an alternative approach of the classical DST to provide better accurate estimation of the grid map occupancy for robot perception.

The idea of using the probabilistic framework to estimate the grid occupancy has been popularized by Elfes in his pioneered works in 1990's ${ }^{9-13}$. Later, the idea has been extended with the fuzzy logic theory framework by Oriolo et al. ${ }^{15-21}$, and in parallel with the belief function (evidential) framework as well ${ }^{22-29}$. Most of the aforementioned research works dealt only with acoustic sensors only (i.e SONAR). Recently, DSmT has also been applied for the perception of the environment with acoustic sensors as reported in. ${ }^{30-32,34-36}$

The authors can be contacted by email at surname.name@onera.fr 
The main contribution of this paper is to propose a new method to make the perception with non acoustic sensors, and to compare the performances of the Proportional Conflict Redistribution rule no. 6 (PCR6) of DSmT with respect to Dempster-Shafer's (DS) rule of combination in terms of accuracy of grid map estimation. In our application, we work with a LIDAR sensor on board on a robot moving in dynamic environment.

This paper is organized as follows. After a short presentation of the basics of belief functions and rules of their combination based on DST and DSmT in the next section, we will present the inverse sensor models in section 3 with the construction of the basic belief assignments (BBA). In section 4, we present an illustrating scenario for environment perception including a mobile object with a platform equipped with a LIDAR, and we compare our new realistic simulation results with those obtained by the probabilistic and the classical beliefbased approaches. We will show how static and mobile objects are extracted from the occupancy grid map using digital image processing. Finally, conclusion and outline perspectives are given in section 5 .

\section{EVIDENTIAL FRAMEWORK}

Dempster-Shafer's theory (DST) of evidence has been developed by Shafer in 1976 from Dempster's works ${ }^{6}$. DST is known also as the theory of belief functions and it is mainly characterized by a frame of discernment (FoD), sources of evidence represented by basic belief assignment (BBA), belief (Bel) and plausibility (Pl) functions, and the Dempster's rule, denoted as DS rule in the sequel* of combination. DST has been modified and extended into Dezert-Smarandache theory ${ }^{8}$ (DSmT) to work with quantitative or qualitative BBA and to combine the sources of evidence in a more efficient way thanks to new proportional conflict redistribution (PCR) fusion rules $-\operatorname{see}^{37-40}$ for discussion and examples. We briefly recall in the next subsections the basics of the theory of belief functions.

\subsection{Belief functions}

Let's consider a finite discrete FoD $\Omega=\left\{\omega_{1}, \omega_{2}, \ldots, \omega_{n}\right\}$, with $n>1$, of the fusion problem under consideration and its fusion space $G^{\Omega}$ which can be chosen either as the power-set $2^{\Omega}$, the hyper-power set ${ }^{\dagger} D^{\Omega}$, or the superpower set $S^{\Omega}$ depending on the model that fits with the problem. ${ }^{8}$ A BBA associated with a given source of evidence is defined as the mapping $m():. G^{\Omega} \rightarrow[0,1]$ satisfying $m(\emptyset)=0$ and $\sum_{A \in G^{\Omega}} m(A)=1$. The quantity $m(A)$ is called mass of belief of $A$ committed by the source of evidence. Belief and plausibility functions are defined by

$$
\operatorname{Bel}(A)=\sum_{\substack{B \subseteq A \\ B \in G^{\Omega}}} m(B) \quad \text { and } \operatorname{Pl}(A)=\sum_{\substack{B \cap A \neq \emptyset \\ B \in G^{\Omega}}} m(B)
$$

The degree of belief $\operatorname{Bel}(A)$ given to a subset $A$ quantifies the amount of justified specific support to be given to $\mathrm{A}$, and the degree of plausibility $\operatorname{Pl}(A)$ quantifies the maximum amount of potential specific support that could be given to A. If for some $A \in G^{\Omega}, m(A)>0$ then $A$ is called a focal element of the BBA $m($.$) .$ When all focal elements are singletons and $G^{\Omega}=2^{\Omega}$ then the BBA $m($.$) is called a Bayesian \mathrm{BBA}^{6}$ and its corresponding belief function $\operatorname{Bel}($.$) is homogeneous to a (possibly subjective) probability measure, and one has$ $\operatorname{Bel}(A)=P(A)=P l(A)$, otherwise in general one has $\operatorname{Bel}(A) \leq P(A) \leq P l(A), \forall A \in G^{\Omega}$. The vacuous BBA representing a totally ignorant source is defined as $m_{v}(\Omega)=1$.

\subsection{Fusion rules}

Many mathematical rules have been proposed in the literature over the decades (see, ${ }^{8}$ Vol. 2 for a detailed list of fusion rules) to combine efficiently several distinct sources of evidence represented by the BBA's $m_{1}(),. m_{2}($.$) ,$ $\ldots, m_{s}().(s \geq 2)$ defined on same fusion space $G^{\Omega}$. In this paper, we focus only on DS rule because it has been historically proposed in DST and it is still widely used in applications, and on the PCR rule no. 6 (i.e. PCR6) proposed in DSmT because it provides a very interesting alternative of DS rule, even if PCR6 is more complex to implement in general than DS rule.

\footnotetext{
*DS acronym standing for Dempster-Shafer since Dempster's rule has been widely promoted by Shafer in the development of his mathematical theory of evidence ${ }^{6}$.

${ }^{\dagger}$ which corresponds to a Dedekind's lattice, see ${ }^{8}$ Vol. 1.
} 
In DST framework, the fusion space $G^{\Omega}$ equals the power-set $2^{\Omega}$ because Shafer's model of the frame $\Omega$ is assumed, which means that all elements of the FoD are exhaustive and exclusive. The combination of the BBA's $m_{1}($.$) and m_{2}($.$) , is done by : m_{1,2}^{D S}(\emptyset)=0$ and for all $X \neq \emptyset$ in $2^{\Omega}$

$$
m_{1,2}^{D S}(X) \triangleq \frac{1}{1-m_{1,2}(\emptyset)} \sum_{\substack{X_{1}, X_{2} \in 2^{\Omega} \\ X_{1} \cap X_{2}=X}} \prod_{i=1}^{2} m_{i}\left(X_{i}\right)
$$

where the numerator of (2) is the mass of belief on the conjunctive consensus on $X$. The denominator $1-m_{1,2}(\emptyset)$ is a normalization constant, where the total degree of conflict denoted $m_{1,2}(\emptyset)$ between the two sources of evidences is defined by

$$
m_{1,2}(\emptyset) \triangleq \sum_{\substack{X_{1}, X_{2} \in 2^{\Omega} \\ X_{1} \cap X_{2}=\emptyset}} \prod_{i=1}^{2} m_{i}\left(X_{i}\right)
$$

According to Shafer ${ }^{6}$, the two sources are said in total conflict if $m_{1,2}(\emptyset)=1$. In this case the combination of the sources by DS rule cannot be done because of the mathematical $0 / 0$ indeterminacy. The vacuous BBA $m_{v}(\Omega)=1$ is a neutral element for DS rule. This rule is commutative and associative, and the formula (2) can be easily generalized for the combination of $s>2$ sources of evidences. DS rule remains the milestone fusion rule of DST.

The doubts of the validity of DS rule has been discussed by Zadeh in $1979^{47-49}$ based on a very simple example with two highly conflicting sources of evidences. Since 1980's, many criticisms have been done about the behavior and the justification of such DS rule. More recently, Dezert et al. in ${ }^{37,38}$ have put in light other counter-intuitive behaviors of DS rule even in low conflicting cases and showed serious flaws in logical foundations of DST. ${ }^{39}$ To overcome the limitations and problems of DS rule of combination, a new family of PCR rules have been developed in DSmT framework. We present the most elaborate one, i.e. the PCR6 fusion rule, which has been used in our perception application for grid occupancy estimation.

In PCR rules, instead of following the DS normalization (the division by $1-m_{1,2}(\emptyset)$ ), we transfer the conflicting mass only to the elements involved in the conflict and proportionally to their individual masses, so that the specificity of the information is entirely preserved. The general principle of PCR consists: 1) to apply the conjunctive rule, 2) to calculate the total or partial conflicting masses; 3) then redistribute the (total or partial) conflicting mass proportionally on non-empty sets according to the integrity constraints one has for the frame $\Omega$. Because the proportional transfer can be done in different ways, there exist several versions of PCR rules of combination. PCR6 fusion rule has been proposed by Martin and Osswald in ${ }^{8}$ Vol. 2, Chap. 2, as a serious alternative to PCR5 fusion rule proposed originally by Smarandache and Dezert in ${ }^{8}$ Vol. 2, Chap. 1. Martin and Osswald had proposed PCR6 based on intuitive considerations and they had shown through different simulations that PCR6 was more stable than PCR5 in term of decision for combining $s>2$ sources of evidence. When only two sources are combined, PCR6 and PCR5 fusion rules coincide, but they differ as soon as more than two sources have to be combined altogether. Recently, it has been proved in ${ }^{40}$ that only PCR6 rule is consistent with the averaging fusion rule which allows to estimate the empirical (frequentist) probabilities involved in a discrete random experiment.

For Shafer's model of FoD ${ }^{\ddagger}$, the PCR6 combination of two BBA's $m_{1}($.$) and m_{2}($.$) is defined by m_{1,2}^{P C R 6}(\emptyset)=0$ and for all $X \neq \emptyset$ in $2^{\Omega}$

$$
m_{1,2}^{P C R 6}(X)=\sum_{\substack{X_{1}, X_{2} \in 2^{\Omega} \\ X_{1} \cap X_{2}=X}} m_{1}\left(X_{1}\right) m_{2}\left(X_{2}\right)+\sum_{\substack{Y \in 2^{\Omega} \backslash\{X\} \\ X \cap Y=\emptyset}}\left[\frac{m_{1}(X)^{2} m_{2}(Y)}{m_{1}(X)+m_{2}(Y)}+\frac{m_{2}(X)^{2} m_{1}(Y)}{m_{2}(X)+m_{1}(Y)}\right]
$$

where all denominators in (4) are different from zero. If a denominator is zero, that fraction is discarded. All propositions/sets are in a canonical form. ${ }^{8}$ Very basic Matlab codes of PCR rules can be found in ${ }^{8,41}$ and from the toolboxes repository on the web. ${ }^{46}$ Like the averaging fusion rule, the PCR6 fusion rule is commutative but not associative. The vacuous belief assignment is a neutral element for this rule.

\footnotetext{
${ }^{\ddagger}$ that is when $G^{\Omega}=2^{\Omega}$, and assuming all elements exhaustive and exclusive.
} 


\subsection{Discounting}

A discounting effect can be applied on a mass function $m($.$) if a piece of information has its reliability lowered.$ In this case, a new mass function $m_{\alpha}($.$) , (with \alpha \in[0,1]$ ) is computed from $m($.$) and a part of the mass of each$ element of the FoD is transferred to the whole $\mathrm{FoD} \Omega$ which represents the total ignorance.

$$
m_{\alpha}(A)= \begin{cases}(1-\alpha) \cdot m(A) & \text { if } A \neq \Omega \\ (1-\alpha) \cdot m(A)+\alpha & \text { if } A=\Omega\end{cases}
$$

\subsection{Pignistic transformation}

Finally, the pignistic transformation $B e t P^{45}$ allows to compute a probability measure from a mass function by distributing proportionally the mass of the subsets on their focal elements:

$$
\forall A \in \Omega, \operatorname{BetP}(A) \triangleq \sum_{B \in 2^{\Omega}} \frac{|A \cap B|}{|B|} \cdot m(B)
$$

where $|A \cap B|$ is the cardinal of the subset $A \cap B$, and $|B|$ is the cardinal of subset the $B$.

However, this transformation is not bijective (a part of the information is lost). So, one can find an infinity of mass functions with the same pignistic probability. This issue is inherent in the nature of probabilities which are not able to distinguish randomness from (epistemic) uncertainty.

\section{EVIDENTIAL OCCUPANCY GRID}

The basic idea of an Occupancy Grid (OG) is to divide the surrounding environment (the ground plane of 2D world) into a set a cells (denoted $C^{i}, i \in[0, n]$ ) in order to estimate their occupancy state. In a probabilistic framework, the aim is to estimate the probabilities $P\left(O^{i} \mid z_{1: t}\right)$ and $P\left(F^{i} \mid z_{1: t}\right)$ given a set of measures $z_{1: t}$ from the beginning up to the current time $t . O^{i}$ (resp. $F^{i}$ ) denotes the occupied (resp. free) state of the cell $C^{i}$. Finally, a decision rule is applied in order to select the most likely state for each cell.

For Evidential approach, occupancy grid represents the information using a mass function over the frame of discernment (FoD) $\Omega=\{F, O\}$. So the mass functions used in grid have the structure

$$
m_{t}=\left[\begin{array}{llll}
m_{t}(\emptyset) & m_{t}(F) & m_{t}(O) & m_{t}(\Omega)
\end{array}\right]
$$

The occupancy mass function can be used during the fusion process, then the decision can be taken using pignistic transform to get a probability measure and use the same decision rule. An interesting part of evidential occupancy grid is that the FoD can be more complex, and as the fusion is done cell by cell the fusion scheme will be still valid .

Occupancy grids can be classified into two categories depending on the use of a forward, or inverse, sensor model. The forward model relies on Bayes inference. Since this approach takes into account the conditional dependency of the cells of the map, it is well adapted to a sensor that observes a large domain of cells with only one reading measurement (e.g. a ultrasonic SONAR). However, it requires heavy processing that can be handled by optimized approximation ${ }^{42}$ or GPU computing. ${ }^{43}$

The inverse model approach is well adapted to narrow fields of measures sensors (e.g. LIDAR). It is composed of two separate steps. First, a snapshot map of the sensor reading is built using an inverse sensor model $P\left(O^{i} \mid z_{t}\right)$. This model can take into account the conditional dependency between the sensor reading and the occupancy of the seen cells. Then, a fusion process (denoted $\odot$ ) is done with the previous map $P\left(O^{i} \mid z_{1: t-1}\right)$ as an independent opinion poll fusion:

$$
P\left(O^{i} \mid z_{1: t}\right)=P\left(O^{i} \mid z_{t}\right) \odot P\left(O^{i} \mid z_{1: t-1}\right)
$$

In the probabilistic framework, the usual fusion operation between states $A$ and $B$ coming from independent measurement, use independent opinion poll ${ }^{52}$ : 


$$
P(A) \odot P(B)=\frac{P(A) \cdot P(B)}{P(A) \cdot P(B)+(1-P(A)) \cdot(1-P(B))}
$$

Inverse approaches have very efficient implementations (e.g. log-odd) that make them popular in mobile robotics. ${ }^{10,14,44}$ Maps built using inverse models are usually less accurate, since they just take into account the dependency of the cells observed in one reading, but it is a good approximation with accurate and high resolution sensors observing a limited number of cells at a time. Moreover, when the sensor is multi-echo and multi-layer, the conditional dependency of the seen cells can be modeled in an efficient way.

\subsection{Fusion strategy with the inverse model}

When dealing with the inverse model approach, an estimate of the pose of the robot has to be available and map grid $G^{M}$ has to be handled. This grid is defined in a world-referenced frame (so it does not move with the robot) and is updated when a new sensor reading is available. Because of the likely evolution of the world in a dynamic environment, the OG update has to be completed by a remanence strategy. The fusion architecture follows then a prediction-correction paradigm and can be used to fuse one or several sensors observations.

Prediction step The prediction step computes the predicted map grid at time $t$ from the map grid estimated at time $t-1$. Depending on the available information, this step can be very refined like done in. ${ }^{43}$ As we consider here that no specific information on the velocity of the objects (or cells) is available, the prediction is done by discounting. The confidence in aged data is controlled by a remanence factor $\alpha \in[0 ; 1]$. The prediction stage is therefore governed by

$$
G_{t}^{M}=\operatorname{discount}\left(\hat{G}_{t-1}^{M}, \alpha\right)
$$

Correction step The correction step consists in the combination of the previously estimated map grid with the grid built from the current measures thanks to the inverse model sensor (see more details $\mathrm{in}^{4,5}$ ). This one is called ScanGrid $G_{t}^{S}$. As this information is referenced in the sensor frame, a $2 \mathrm{D}$ warping is applied to reshape this grid into the fusion frame. To perform this operation, the current pose $q_{t}$ is estimated using a GPS sensor and the rigid homogeneous transformation matrix $H_{t}$ is computed. When GPS becomes unavailable, the CAN (Controller Area Network) bus is used to get the robot odometric data. The motion matrix $H_{t}$ and the extrinsic calibration matrix $C$ are used to compute a remapping function $f(x . y)$ according to Eq.(11) below

$$
f(x, y)=C \cdot H_{t} \cdot\left[\begin{array}{l}
x \\
y \\
1
\end{array}\right]
$$

Finally, the ScanGrid is remapped with $f$ and fused with the previous map grid.

$$
G_{t}^{M}(i, j)=\hat{G}_{t}^{M}(i, j) \odot G_{t}^{S}(f(i, j))
$$

The grid $G_{t}^{S}$ represents the BBA produced by the sensor model. This BBA is created in respect to sensor data (e.g. LIDAR point here) and a sensor model to infer an instant occupancy grid. For probabilistic approach, it refers to the occupancy probability $P_{t}^{S}(O)$, for evidential approach it refers for a occupancy mass function $m_{t}^{S}=\left[\begin{array}{llll}m_{t}^{S}(\emptyset) & m_{t}^{S}(F) & m_{t}^{S}(O) & m_{t}^{S}(\Omega)\end{array}\right]$. The grid $\hat{G}_{t}^{M}$ refers to the previous MapGrid $G_{t-1}^{M}$ predicted at current time using Eq.(10). In the following parts, for each approach considered, the rule $\odot$ used in Eq.(12) is different. Bayesian approach uses Eq.(9), DS approach uses Eq.(2) and PCR6 approach uses Eq.(4). 


\subsection{Discounting in Occupancy Grids}

The main advantage of using discounting is to provide a simple way to model the presence of dynamic object in the scene. This model allows to make a prediction without information on the dynamic at the cell level (or at the object level) which is generally not directly available from sensors and merely difficult to estimate without greedy time-computing algorithms ${ }^{43}$ (especially when the evidential framework is adopted). The main issue with the discounting effect is that it makes impossible to build persistent static map. Indeed, cells not viewed by the sensor will quickly converge to the ignorance state. Therefore, this strategy cannot be used to build the map of a building for instance. If we are interested to build static map in presence of moving objects, the discounting function is then not recommended. We will see why in the next part of the paper where in this case Bayesian and DS fusion rules will not be very efficient. To handle this case, we will show why it is recommended to use the PCR6 rule.

\section{RESULTS}

In this section, we present simulation results of grid occupancy estimation in a realistic scenario based on different rules of combination (Bayesian fusion, Dempster-Shafer rule, and PCR6 fusion rule).

\subsection{Basic simulation}

Setup: In order to present the basic behavior of the different combination rules studied, we have realized at first some simple 1D-simulations, where we consider a grid cell crossed by a moving object. In this case, the state of the cell changes from free-state to occupied-state at time $t_{1}$ and from occupied-state to free-state at time $t_{2}$. Figure 1 shows the results of these simulations under different conditions. On each subfigure, we show on the top plot the real state of the cell (i.e. the groundtruth). The second raw of each subplot shows the sensor data simulated that corresponds to the BBA of the state of the cell. This mass function is built according to the state of the cell, the level of confidence of the sensor and can be eventually perturbed with additional noises. FA indicates the rate of False Alarms, and ND the rate of Non Detections. We will consider different levels of confidence for $m_{S G}(O)$ when the cell is occupied, and $m_{S G}(F)$ when the cell if free. The bottom plot of each subplot represents the level of belief of the cell state obtained with Bayesian fusion, Dempster-Shafer (DS) fusion and the PCR6 fusion rules respectively.

Effect of discounting: Figure 1a presents the results of the classical chain using a discounting factor $\alpha=0.05$ while figure $1 \mathrm{~b}$ presents the same case without discounting $(\alpha=0)$. If the discounting is applied, all the fusion rules behave similarly, but if the discounting is not used, a lag appears with Bayesian and DS fusion rules. The lag effect is seriously reduced with PCR6 rule.

Performances analyses: The performance of our method is summarized in Table 1. For each simulation, 10000 Monte Carlo runs have been performed, in order to estimate the false alarm and non detection rates. In order to make the decision, the pignistic probability has been computed and a MAP estimator has been used. For each simulation presented in the Table 1, we also mention the discounting level, the rate of noise impacting sensor observations.

Simulations 0 and 1, illustrated by the figure 1a and the figure 1b, correspond to the noise-free situation. By removing the discounting operator, Bayesian and DS approach have a lag in the detection of the change of state that impacts clearly their performances. The PCR6 approach is much less concerned by this effect because of the proportional conflict redistribution process. Simulations 2 and 3 (see figure 1c) include $10 \%$ of wrong measurement caused by noises. The fusion rules behave similarly as for simulation 0 and 1 , but the performances are a bit lower which reflects the effect of noisy measurements in the grid estimation process. For simulations 4, 5 and 6 , the noise reaches $15 \%$ for ND and $30 \%$ for FA which is quite strong. As we see in figure $1 \mathrm{~d}$, the Bayesian and DS fusion rules are not able to detect the second state change, during the simulation time. This induces bad False alarm rates. In the last simulations 7 and 8, the noise is about $25 \%$ of ND and $50 \%$ of FA. In these conditions, all the methods have poor false alarm rates but the PCR6 keeps good (low) non detection rates. 

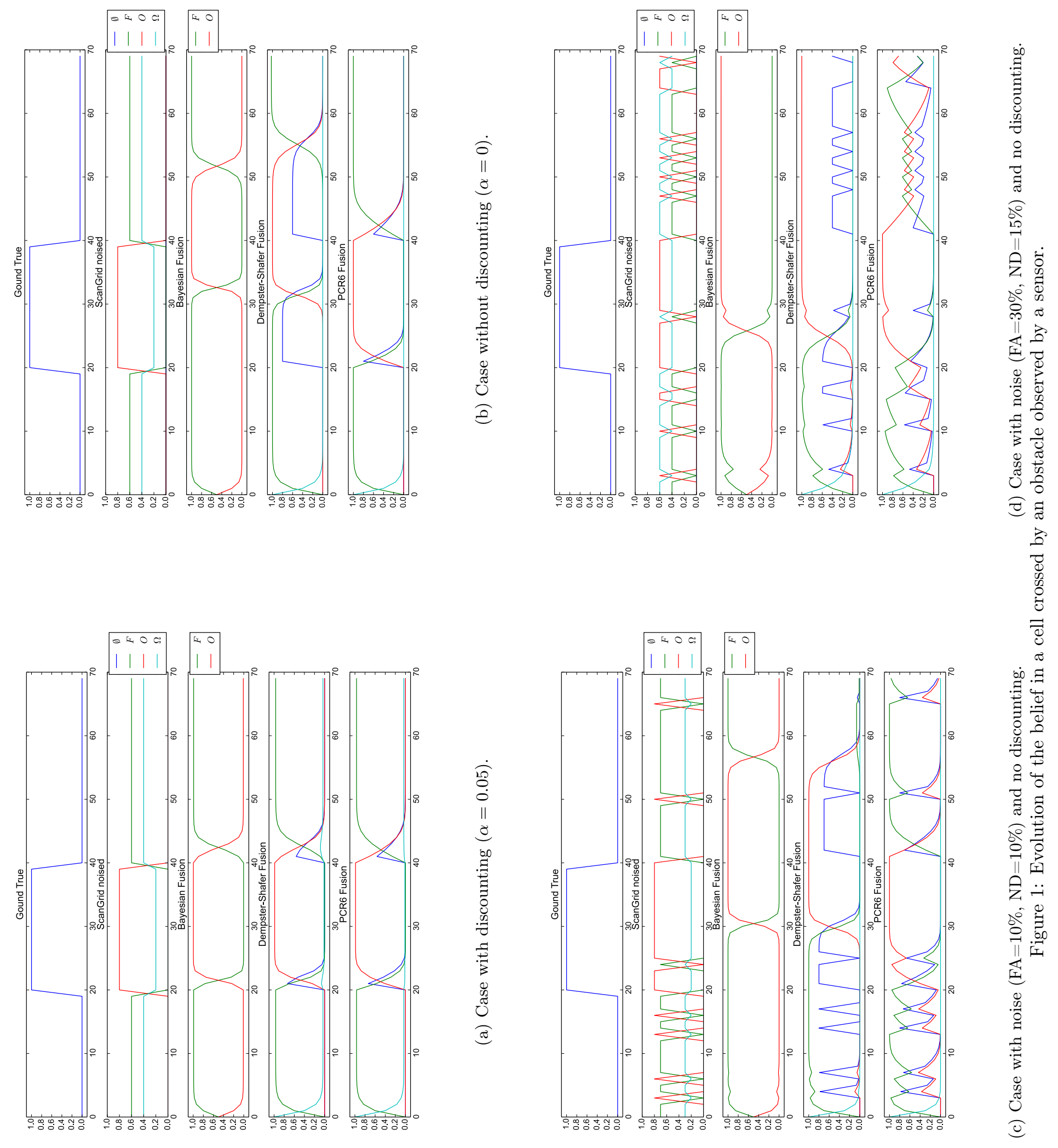


\begin{tabular}{|c|c|c|c|c|c|c|c|c|c|c|}
\hline $\mathrm{N}^{\circ}$ & discounting & key time & sensor noise & sensor belief & \multicolumn{2}{|c|}{ Bayesian } & \multicolumn{2}{|c|}{ DS } & \multicolumn{2}{|c|}{ PCR6 } \\
\hline & $\alpha$ & $t_{1} / t_{2}$ & $N D / F A$ & $m_{S G}(O) / m_{S G}(F)$ & ND & FA & $\mathrm{ND}$ & FA & $\mathrm{ND}$ & FA \\
\hline 0 & 0.05 & $20 / 40$ & 0 & $0.8 / 0.6$ & 10.0 & 6.0 & 10.0 & 6.0 & 10.0 & 6.0 \\
\hline 1 & 0 & $20 / 40$ & 0 & $0.8 / 0.6$ & 65.0 & 24.0 & 60.0 & 32.0 & 10.0 & 0.6 \\
\hline 2 & 0.05 & $20 / 40$ & $10 / 10$ & $0.8 / 0.6$ & 11.2 & 9.2 & 10.5 & 10.0 & 10.0 & 9.6 \\
\hline 3 & 0 & $20 / 40$ & $10 / 10$ & $0.8 / 0.6$ & 77.7 & 15.2 & 73.5 & 18.9 & 11.5 & 6.7 \\
\hline 4 & 0.05 & $20 / 40$ & $15 / 30$ & $0.8 / 0.6$ & 9.2 & 28.0 & 8.2 & 31.5 & 8.4 & 28.9 \\
\hline 5 & 0 & $20 / 40$ & $15 / 30$ & $0.8 / 0.6$ & 33.0 & 62.7 & 26.9 & 65.8 & 8.4 & 28.8 \\
\hline 6 & 0 & $20 / 40$ & $15 / 30$ & $0.6 / 0.4$ & 31.3 & 63.9 & 26.0 & 67.3 & 9.3 & 38.7 \\
\hline 7 & 0 & $20 / 40$ & $25 / 50$ & $0.6 / 0.4$ & 15.1 & 76.9 & 11.5 & 79.4 & 5.7 & 64.0 \\
\hline 8 & 0 & $20 / 40$ & $25 / 50$ & $0.4 / 0.2$ & 7.1 & 83.9 & 5.1 & 85.1 & 1.9 & 87.3 \\
\hline
\end{tabular}

Table 1: Comparison of false alarm and non detection rates (\%).

\subsection{LIDAR simulation}

In this simulation, the DS and PCR6 fusion rules are compared on a 2D occupancy grid problem close to real application for robot perception. The simulation was realized using the Robot Operating System (ROS) ${ }^{50}$ environment and the Gazebo ${ }^{51}$ simulator is used here to simulate a Hokuyo LIDAR and a moving object as shown on Figure 2a. The simulated sensor has a FoV (Field of View) about $270^{\circ}$ and a max range about $10 \mathrm{~m}$. The rate of the scan is $20 \mathrm{~Hz}$ and the ranges of the LIDAR point are corrupted with a Gaussian noise $\mathcal{N}(0,0.1)$.

Figure $2 \mathrm{~b}$ shows a simulated LIDAR scan. The beams that do not hit obstacle within the range are considered as max range (as done in the real Hokuyo sensor). The moving object is a box which has a circular trajectory and moves at $6 \mathrm{rpm}$ around the LIDAR. A ground true grid is computed according the real position of the box and its geometry at each scan time. The grid used is a square of $10 \mathrm{~m}$ by $10 \mathrm{~m}$ with a resolution of $0.1 \mathrm{~m}$, and the ScanGrid BBA are set to $m_{S G}(O)=0.8, m_{S G}(\Omega)=0.2$ for occupied cells and $m_{S G}(F)=0.6, m_{S G}(\Omega)=0.4$ for free cells.

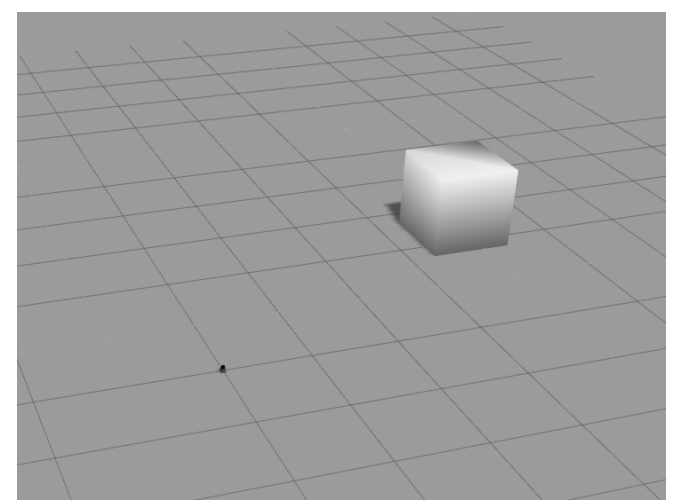

(a) View of the Gazebo simulation: the box turns around the LIDAR sensor.

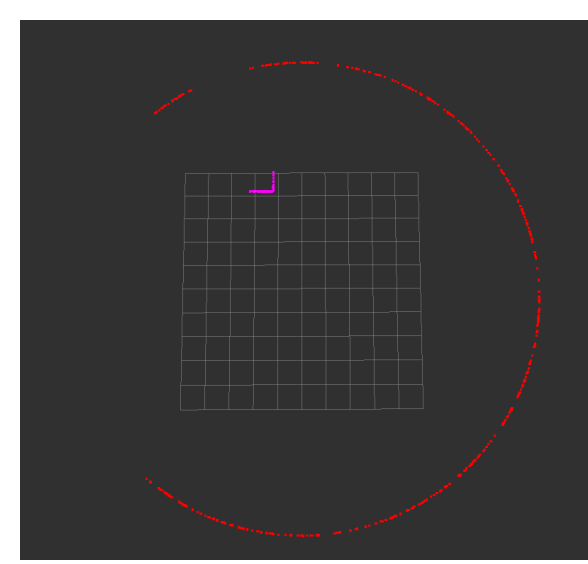

(b) Bird view of one LIDAR scan.

Figure 2: Simulation setup.

In order to quantify the results, we compute some metrics. However, because of occlusion, only the cells located on the edges of the box can be considered, that is why we don't consider global metrics. We consider here the two following metrics: 1) the number of correct occupied cell (proportional to recall in our case), and 2) the number of conflicting cells close to the box. The first describes the ability of the method to add objects into the map and also by analogy to remove object from the map. The second describes the ability of the method 
to detect moving objects by generating conflict. This ability is important and is one of the improvement of evidential grid against classical Bayesian grid estimation.

Figures 3 and 4 show the result over one turn. The number of cells detected for both metrics depends a lot on the position around the sensor. This can be explained because, in some place, the LIDAR sensor is able to see two edges of the box. In other situations, the LIDAR sensor detects just one edge, and when the box is behind (on the back of) the sensor it is out of the field of view of the LIDAR. On figure 3, we can see that the number of occupied cells with the PCR6 fusion is greater than with the DS fusion. Contrarily to the PCR6, the DS fusion without discounting cannot estimate well the quick changes of states in the map. From the motion standpoint, figure 4 shows that the PCR6 approach keeps the same level to generate conflict in presence of moving object (similar to DS fusion). The x-axis of Figures 3 and 4 is the time stamp of the LIDAR scans, and the y-axis is the number of cells in different states (occupied for Fig. 3, or with conflict for Fig. 4).

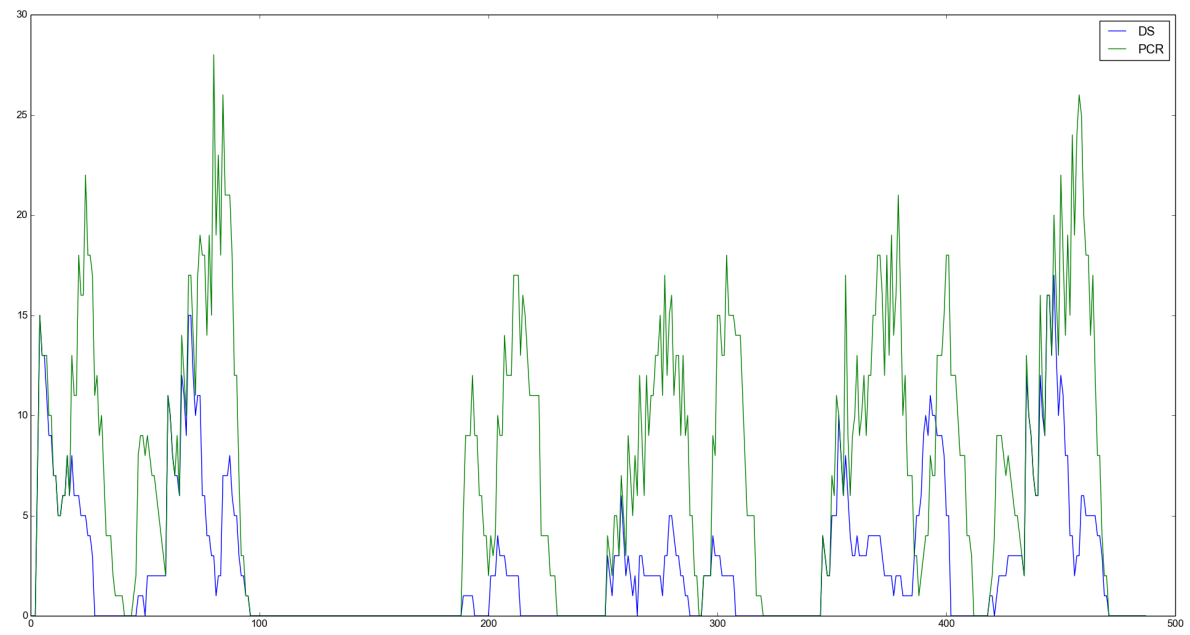

Figure 3: 2D LIDAR simulation: Number of correct occupied cells (green=PCR6,blue=DS).

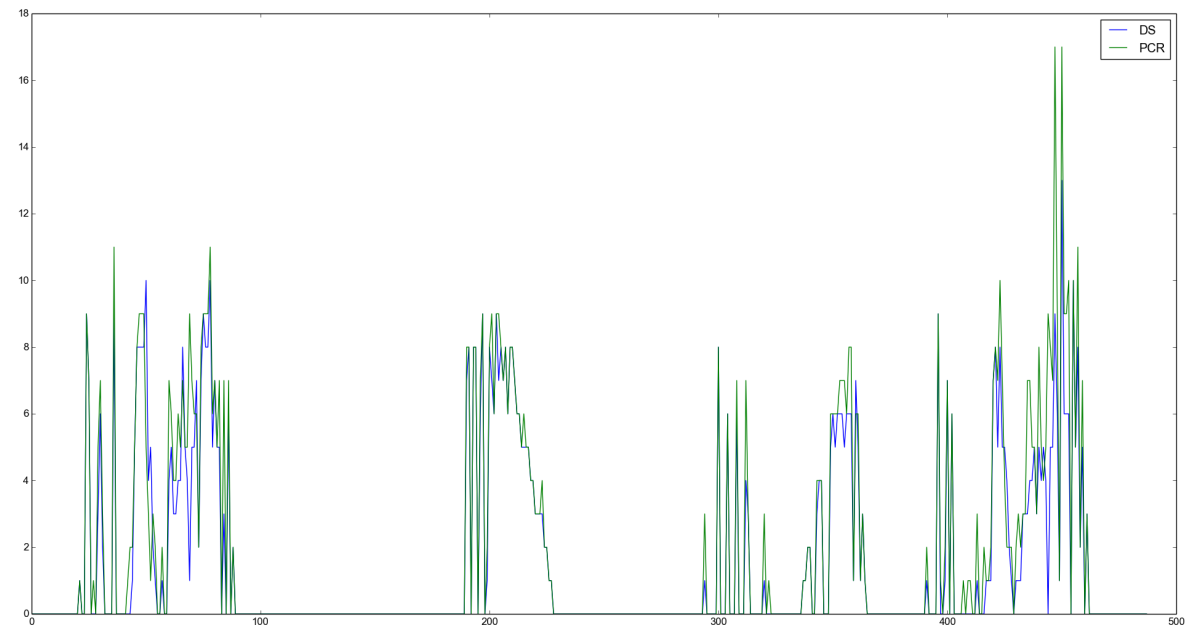

Figure 4: 2D LIDAR simulation: Number of conflicted cells into the box shape (green=PCR6,blue=DS). 


\subsection{Real data processing}

A real experimentation was realized using an Hokuyo UTM-30LX sensor. This experimentation takes place in an office in which a person was walking into. The evidential occupancy grid fusion node was implemented within the ROS environment. The grid has the same size and resolution as in the previous example. The BBA used in the sensor model has been set to $m_{S G}(O)=0.8, m_{S G}(\Omega)=0.2$ for occupied cells and $m_{S G}(F)=0.86, m_{S G}(\Omega)=0.2$ for free cells. No discounting was applied.

Figure 5 presents the occupancy grid estimation using DS and PCR6 rules of combination and for two typical snapshots of the sequence. The color of cells denotes the state having the highest mass value: green for $F$ (free state), red for $O$ (occupied state), and black for $\Omega$ (full uncertainty). For convenience, we have also displayed in blue all the cells that carry a conflicting mass $m(\emptyset)>0.1$ before applying the normalization step of DS rule, or before applying the proportional conflict redistribution with PCR6. Figure 5a and 5c show the result using DS rule. The room scanned by the sensor is correctly mapped and its bounds (mainly walls and doors) are clearly identified by the red pixels. The free space (green pixels) is correctly detected in the room except near the people that is labeled as free (with conflicting cell shown in blue for convenience). The people moving around the desk in the office room is only detected from conflicting cells when he stops to walk several times. Figure $5 \mathrm{~b}$ and $5 \mathrm{~d}$ show the PCR6 result at the same time stamps. In this case, the people is correctly detected as shown by the red pixels (occupied cells) inside the green area (the office room). A conflict cell is created when he starts walking in the room. The static part of the room is also detected (as with DS fusion rule).

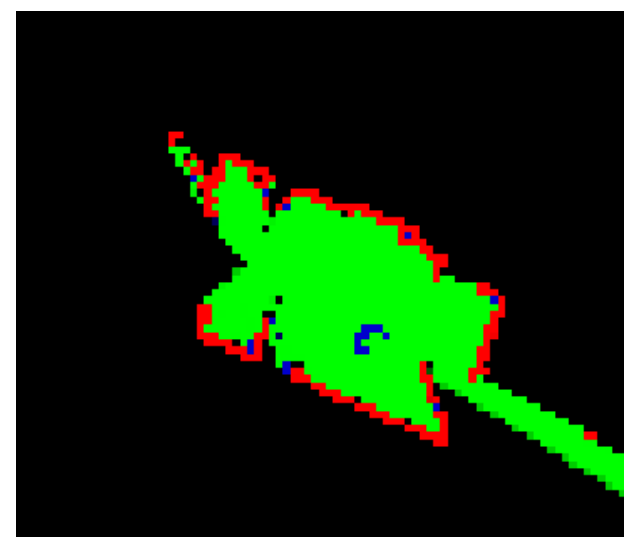

(a) Snapshot 1 - DS fusion.

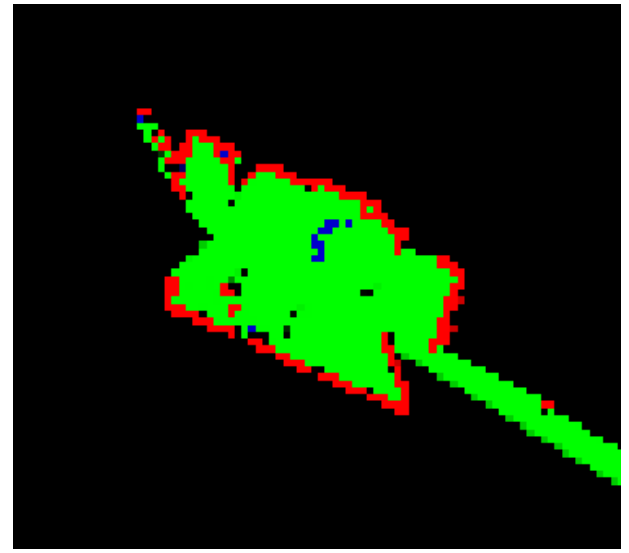

(c) Snapshot 2 - DS fusion.

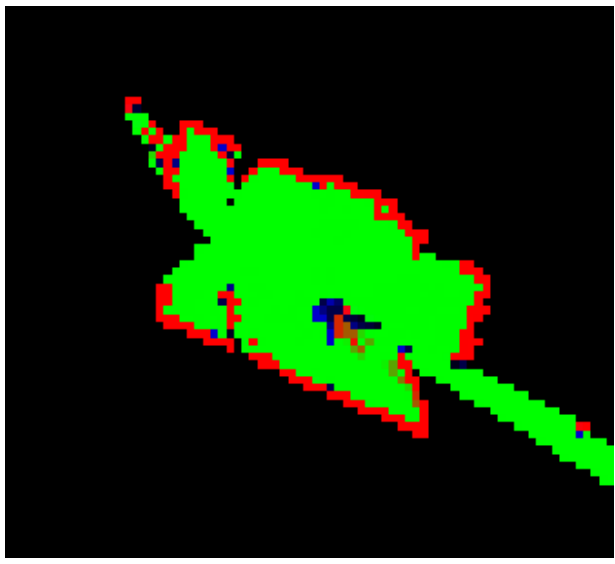

(b) Snapshot 1 - PCR6 fusion.

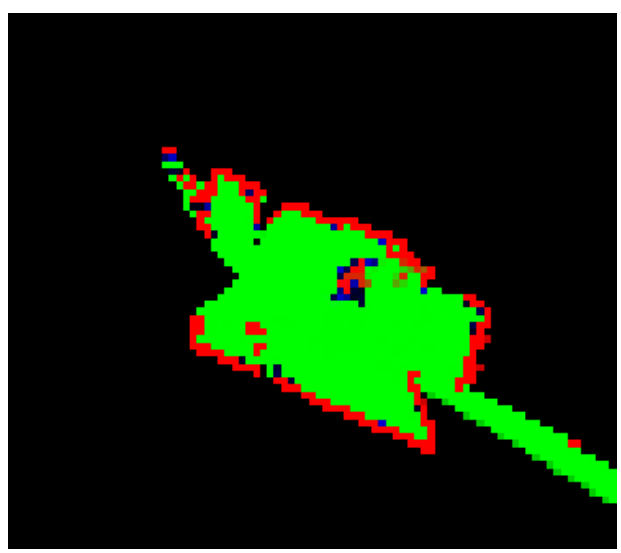

(d) Snapshot 2 - PCR6 fusion.

Figure 5: Result of evidential occupancy grid in real experimentation. 


\section{CONCLUSIONS AND PERSPECTIVES}

In this work we have presented a novel application of the belief functions which significantly improves the map building process for robots environment perception and grid map estimation. This work shows the importance of defining an accurate sensor model. We have considered the uncertainties of the LIDAR measurements and used the PCR6 rule of DSmT to model and combine sensor information. Our new method differs of Bayesian approach by allowing support for more than one proposition at a time, rather than a single hypothesis. It is a intervalbased approach, as defined by the lower and upper probability bounds [Bel, Pl] allowing the lack of measurement to be modeled adequately. This new method differs also from the classical evidential approach because the PCR6 rule is used instead of DS rule. Experimental results with the LIDAR confirm the improvements of the accuracy of this new grid estimation method with respect to previous methods. As perspectives, we will try to implement this fusion rule in 3D occupancy grid (Octomap based) and use a stereo camera with dense disparity map computation as sensor source. In future works, we will consider in this perception context more classes into the frame of discernment and we will also test the improved PCR6 rule of combination including Zhang's degree of intersection.

\section{REFERENCES}

1. P.S.P. Stepan, M.K.M. Kulich, L.P.L. Preucil, Robust data fusion with occupancy grid, IEEE Trans. on SMC, Part C, Vol. 35, No. 1, 2005.

2. H. Zhao, et al. , SLAM in a dynamic large outdoor environment using a laser scanner, in 2008 IEEE Int. Conf. on Robotics and Automation, pp. 1455-1462, May 2008.

3. A. Cherubini, F. Chaumette, Visual navigation with obstacle avoidance, in 2011 IEEE/RSJ Int. Conf. on Intelligent Robots and Systems, pp. 1593-1598, Sept. 2011.

4. J. Moras, V. Cherfaoui, P. Bonnifait, Credibilist occupancy grids for vehicle perception in dynamic environments, 2011 IEEE Int. Conf on Robotics and Automation, Shanghai, China, pp. 84-89, May 2011.

5. J. Moras, V. Cherfaoui, P. Bonnifait, Moving Objects Detection by Conflict Analysis in Evidential Grids, 2011 IEEE Intelligent Vehicles Symposium IV, pp. 1122-1127, June 2011.

6. G. Shafer, A Mathematical Theory of Evidence. Princeton: Princeton University Press, 1976.

7. J. Moras, V. Cherfaoui, P. Bonnifait, Evidential Grids Information Management in Dynamic Environments, Proc. of Fusion 2014 Int. Conf, Salamanca, Spain, July 2014.

8. F. Smarandache, J. Dezert, Advances and applications of DSmT for information fusion (Collected works), American Research Press, USA Vol.1, 2, 3 \& 4, 2004-2015. http://www.onera.fr/staff/jean-dezert?page=2

9. A. Elfes, Occupancy Grid: A Probabilistic Framework for Robot Perception and Navigation, Ph.D. Thesis, Carnegie Mellon University, PA, USA, 1989.

10. A. Elfes, Using occupancy grids for mobile robot perception and navigation, Computer, Vol. 22, no. 6, pp. 46-57, 1989.

11. A. Elfes, Occupancy grids: A Stochastic spatial representation for active robot perception, in Proc. of the Conference on Uncertainty in AI, pp. 60-70, 1990.

12. A. Elfes, Occupancy grids: A stochastic spatial representation for active robot perception, in Autonomous Mobile Robots: Perception, Mapping, and Navigation (S. S. Iyengar and A. Elfes, eds.), IEEE Computer Society Press, pp. 60-71, 1991.

13. A. Elfes, Multi-source spatial data fusion using Bayesian reasoning, in: M.A. Abidi, R.C. Gonzales (Eds.), Data Fusion in Robotics and Machine Intelligence (Chapter 3), Academic Press, New York, 1992.

14. H.P. Moravec. Sensor fusion in certainty grids for mobile robots. AI Magazine, Summer: 116-121, 1988

15. G. Oriolo, G. Ulivi, M. Vendittelli, On-line map building and navigation for autonomous mobile robots, Proc. 1995 IEEE Int. Conf. on Robotics and Automation, pp. 2900-2906, Nagoya, Japan, 1995.

16. G. Oriolo, G. Uliviy, M. Vendittelli, Fuzzy maps: A new tool for mobile robot perception and planning, Journal of Robotic Systems, Vol. 14, No. 3, pp. 179-197, 1997.

17. K. Konolige, Improved occupancy grids for map building, Autonomous Robots, Vol. 4, No. 4, pp. 351-367, 1997. 
18. G. Oriolo, G. Ulivi, Real time map building and navigation for autonomous robots in unknown environments, IEEE Trans. on SMC, Vol. 28, No. 3, pp. 316-332, 1998.

19. G. Oriolo, G. Ulivi, M. Vendittelli, Real-time map building and navigation for autonomous robots in unknown environments, IEEE Transactions on SMC, Vol. 5, 1999.

20. S. Noykov, C. Roumenin, Occupancy grids building by SONAR and mobile robot, Robotics and Autonomous Systems, Vol. 55, pp. 162-175, 2007.

21. I-H. Li, C.-C. Hsu, S.-S. Lin, Map Building of Unknown Environment Based on Fuzzy Sensor Fusion of Ultrasonic Ranging Data, International Journal of Fuzzy Systems, Vol. 16, No. 3, pp. 368-377, September 2014.

22. K. Hughes, R. Murphy, Ultrasonic robot localization using Dempster-Shafer theory, in Proc. of SPIE on Neural and Stochastic Methods in Image and Signal Processing, 1992, pp. 2-11, 1992.

23. P. Tirumalai, B.G. Schunk, R.C. Jain, Evidential reasoning for building environment maps, IEEE Trans. on Systems, Man, and Cybernetics, Vol. 25, No. 1, pp. 10-20, 1995.

24. F. Gambino, G. Oriolo, G. Ulivi, A comparison of three uncertainty calculus techniques for ultrasonic map building, in: Proc. 1996 SPIE Int. Symp. on Aerospace/Defense Sensing and Control, pp. 249-260, Orlando, FL, USA, 1996.

25. D. Pagac, E. Nebot, H. Durrant-Whyte, An evidential approach to probabilistic map-building, Proc. of IEEE International Conference on Robotics and Automation, Vol. 1, pp. 745-750, Minneapolis, MN, USA, April 1996.

26. D. Pagac, E. Nebot, H. Durrant-Whyte, An evidential approach to map-building for autonomous vehicles, IEEE Trans. on Robotics and Automation, Vol. 14, No. 4, pp. 623-629, 1998.

27. M. Ribo, A. Pinz, A comparison of three uncertainty calculi for building SONAR-based occupancy grids, Robotics and Autonomous Systems, Vol. 31, pp. 201-209, 2001.

28. T. Reineking, J. Clemens, Evidential FastSLAM for grid mapping, Proc. of Fusion 2013 Conf., pp. 789-796, Istanbul, Turkey, July 2013.

29. J. Clemens, T. Reineking, Multi-Sensor Fusion Using Evidential SLAM for Navigating a Probe through Deep Ice, Proc. Of Belief 2014, Oxford, UK, Sept. 2014.

30. X. Li, X. Huang, M. Wang, J. Xu, H. Zhang, DSmT Coupling with PCR5 for Mobile Robots Map Reconstruction, Proc. of 2006 Int. Conf. on mechatronics and Automation, Luoyang, China, June 26-28, 2006.

31. X. Li, X. Huang, M. Wang, Robot Map Building from SONAR Sensors and DSmT, Information \& Security : An Int. J., Bulg. Acad. of Sci., Vol. 20, pp. 104-121, Sofia, March 2006.

32. X., Huang, X. Li, J. Dezert, M. Wang, A Fusion Machine Based on DSmT and PCR5 for Robot's Map Reconstruction, Int. Journal of information Acquisition, Vol. 3, No.3, pp. 201-211, Sept. 2006.

33. X. Li, X. Huang, M. Wang, J. Dezert, A fusion Machine based on DSmT and PCR5 for robot's map reconstruction, International Journal of Information Acquisition, Vol. 3, No. 3, pp. 1-11, 2006.

34. X. Li, X. Huang, J. Dezert, L. Duan L., M. Wang, A successful application of DSmT in SONAR grid map building and comparison with DST-based approach, Int. Journal of Innovative Computing, Information and Control, Vol. 3, No. 3, June 2007.

35. P. Li, X. Huang, S. Yang, J. Dezert, SLAM and path planning of mobile robot using DSmT, Journal of Software Engineering, Vol. 7, No. 2, pp. 46-67, 2013.

36. J. Zhou, J. Duan, G. Yang, Occupancy Grid Mapping Based on DSmT for Dynamic Environment Perception, International Journal of Robotics and Automation (IJRA), Vol. 2, No. 4, pp. 129-139, December 2013.

37. J. Dezert, P. Wang, A. Tchamova, On the validity of Dempster-Shafer theory, Proc. of Fusion 2012 Int. Conf., Singapore, July 9-12, 2012.

38. A. Tchamova, J. Dezert, On the behavior of Dempster's Rule of combination and the foundations of Dempster-Shafer theory, (Best paper awards), 6th IEEE Int. Conf. on Int. Syst. (IS '12), Sofia, Bulgaria, Sept. 6-8, 2012.

39. J. Dezert, A. Tchamova, On the validity of Dempster's fusion rule and its interpretation as a generalization of Bayesian fusion rule, International Journal of Intelligent Systems, Special Issue: Advances in Intelligent Systems, Vol. 29, Issue 3, pages 223-252, March 2014. 
40. F. Smarandache, J. Dezert, On the consistency of PCR6 with the averaging rule and its application to probability estimation, Proc. of Fusion 2013, Istanbul, Turkey, July 2013.

41. F. Smarandache, J. Dezert, J.-M. Tacnet, Fusion of sources of evidence with different importances and reliabilities, Proc. of Fusion 2010 Int. Conf., Edinburgh, UK, July 26-29, 2010.

42. S. Thrun, Learning occupancy grid maps with forward sensor models, Autonomous robots, Vol. 15, No. 2, pp. 111-127, 2003.

43. M.K. Tay, K. Mekhnacha, M. Yguel, C. Coué, C. Pradalier, and C. Laugier, The Bayesian occupation filter, in Probabilistic Reasoning and Decision Making in Sensory-Motor Systems (P. Bessière, C. Laugier, and R. Siegwart, eds.), pp. 77-98, Springer Berlin Heidelberg, Springer Ed., 2008.

44. T. Weiss, B. Schiele, K.Dietmayer, Robust Driving Path Detection in Urban and Highway Scenarios Using a Laser Scanner and Online Occupancy Grids, 2007 IEEE Intelligent Vehicles Symposium, pp. 184-189, June 2007.

45. P. Smets, Decision making in the TBM: the necessity of the pignistic transformation, International Journal of Approximate Reasoning, Vol. 38, pp. 133-147, Feb. 2005.

46. http://bfas.iutlan.univ-rennes1.fr/wiki/index.php/Toolboxs

47. L.A. Zadeh, On the validity of Dempster's rule of combination, Memo M79/24, Univ. of California, Berkeley, CA, U.S.A., 1979.

48. L.A. Zadeh, Book review: A mathematical theory of evidence, The Al Magazine, Vol. 5, No. 3, pp. 81-83, 1984.

49. L.A. Zadeh, A simple view of the Dempster-Shafer theory of evidence and its implication for the rule of combination, The Al Magazine, Vol. 7 (2), pp. 85-90, 1986.

50. Morgan Quigley and Ken Conley and Brian P. Gerkey and Josh Faust and Tully Foote and Jeremy Leibs and Rob Wheeler and Andrew Y. Ng, ROS: an open-source Robot Operating System, in 2009 ICRA Workshop on Open Source Software, 2009.

51. Nathan Koenig and Andrew Howard, Design and Use Paradigms for Gazebo, An Open-Source Multi-Robot Simulator, in 2004 IEEE/RSJ International Conference on Intelligent Robots and Systems, pp. 2149-2154, Sept. 2004.

52. Robert A. Jacobs, Methods for combining expert's probability assessments, Neural Computation, Vol. 7 (5), pp. 867-888, 1995 